Article

\title{
Type and Cotype Constants and the Linear Stability of Wigner's Symmetry Theorem
}

\author{
Javier Cuesta ${ }^{1,2}$ (D) \\ 1 Department of Mathematics, Technische Universität München, 85748 Garching, Germany; j.cuesta@tum.de \\ 2 Munich Center for Quantum Science and Technology (MCQST), D-80799 München, Germany
}

Received: 5 August 2019; Accepted: 23 August 2019; Published: 3 September 2019

check for updates

\begin{abstract}
We study the relation between almost-symmetries and the geometry of Banach spaces. We show that any almost-linear extension of a transformation that preserves transition probabilities up to an additive error admits an approximation by a linear map, and the quality of the approximation depends on the type and cotype constants of the involved spaces.
\end{abstract}

Keywords: Wigner's theorem; stability; almost-symmetry; almost-linear; type; cotype; Banach spaces

\section{Introduction and Preliminaries}

In the work of N. J. Kalton [1-3], we can find novel ideas and methods for the stability of functional equations that depart from the classical methods of Hyers, Ulam and Rassias [4]. In Ref. [3] (see Theorem 2.2), Kalton provides a sharp bound on the stability of the additive map in $\mathbb{R}^{n}$ for the so-called singular case. His proof makes use of probabilistic and geometric methods in Banach space theory. This paper ends with a sketch on how the theory of twisted sums in Banach space theory could be used to obtain the same result. In this note, we develop this last idea and use it to obtain an improvement in the linear stability of Wigner's symmetry theorem (see Theorem 3).

Wigner's celebrated symmetry theorem [5] is not only central for physics, but it also finds an important role in many preservers' problems. A preserver problem deals with the characterization of maps, primarily on matrix spaces and operator algebras that preserve certain functional, subset, or an invariant. In particular, in the field of Quantum Information Theory (QIT) it has been shown [6] that the only mapping $T$ that preserves the $f$-divergences (this includes the von Neumann and relative entropy) is a Wigner symmetry transformation, i.e., of the form $T(x)=U x U^{*}$, where $U$ is either a unitary or antiunitary transformation on $\mathbb{C}^{d}$. It turns out that most of the proofs of different preservers problems can be reduced to Wigner's theorem. Therefore, it is natural to expect that sharp bounds on the stability of Wigner's theorem could provide good approximations for a wide range of almost-preserving problems. It is worth pointing out that there exists a close relation between geometric functional analysis and many questions in QIT [7]. This is the point of view that we want to motivate here.

It has been recently shown [8] that an arbitrary almost-symmetry in quantum theory, i.e., a transformation on the set of pure states $\mathbb{P}(\mathcal{H})$ in a separable complex Hilbert space $\mathcal{H}$ that almost preserves the transition probabilities up to an error $\varepsilon$, can be approximated by a linear map $H$ if and only if $\mathcal{H}$ is a finite-dimensional Hilbert space. For an infinite-dimensional Hilbert space, the approximation is in a weak sense (see Theorem 2-(i) in [8]). The quality of the approximation for a $d$-dimensional Hilbert space $\mathcal{H}$ was obtained to be

$$
\frac{1}{3} \sqrt{\frac{\ln (d-1) \varepsilon}{4 \pi}} \leq\|f(x)-H(x)\|_{2} \leq 4 d \sqrt{\varepsilon}
$$

where $\|\cdot\|_{2}$ is the Hilbert-Schmidt norm. The main idea for the upper bound in Equation (1) was to consider an almost-linear extension of $f$ with some particular domain and codomain, followed by 
an application of the geometric Hahn-Banach theorem. In this work, we explore how the quality of the approximation depends on the consideration of various classes of almost-linear extensions. These extensions now have arbitrary finite-dimensional Banach spaces as domain and codomain.

Throughout this note, we will be entirely concerned with finite-dimensional Banach spaces and the twisted sums generated by almost-linear maps. A map $F: X \rightarrow Y$ between Banach spaces will be called almost-linear if it satifies the following two conditions:

(i) $\quad F(\lambda x)=\lambda F(x)$ for all $\lambda \in \mathbb{R}$ and $x \in X$,

(ii) there exists a $\delta>0$ such that for any finite sequence $\left(x_{i}\right)_{i=1}^{m} \subset X, m \in \mathbb{N}$ and $\lambda \in \mathbb{R}^{m}$,

$$
\left\|\sum_{i=1}^{m} \lambda_{i} F\left(x_{i}\right)-F\left(\sum_{i=1}^{m} \lambda_{i} x_{i}\right)\right\|_{Y} \leq \delta \sum_{i=1}^{m}\left|\lambda_{i}\right|\left\|x_{i}\right\|_{X}
$$

We will show that, for every almost-linear map $F$, there exists a linear map $H$ whose distance to $F$ depends additively on $\delta$ and on some geometric invariants of the domain and target space of $F$ (see Theorem 1). The Banach space numbers used to express the results are the type and cotype constants which we introduce now. Let $\left\{\gamma_{j}\right\}_{j=1}^{n}$ be a sequence of independent real Gaussian random variables, i.e., for each Borel subset $B \subset \mathbb{R}$, each random variable has a distribution

$$
\mu(\gamma \in B)=\frac{1}{(2 \pi)^{1 / 2}} \int_{B} e^{-\frac{t^{2}}{2}} d t .
$$

Let $X$ be a Banach space with norm $\|\cdot\|$ and let $p \in[1,2], q \in[2, \infty)$. For every positive interger $n$, we define $T_{p, n}(X), C_{q, n}(X)$ to be the smallest constants such that for arbitrary sequences $\left\{x_{j}\right\}_{j=1}^{n} \subset X$, we have

$$
\begin{array}{r}
\left(\mathbb{E}\left\|\sum_{j=1}^{n} \gamma_{j} x_{j}\right\|^{2}\right)^{1 / 2} \leq T_{p, n}(X)\left(\sum_{j=1}^{n}\left\|x_{j}\right\|^{p}\right)^{1 / p}, \\
C_{p, n}(X)^{-1}\left(\sum_{j=1}^{n}\left\|x_{j}\right\|^{q}\right)^{1 / q} \leq\left(\mathbb{E}\left\|\sum_{j=1}^{n} \gamma_{j} x_{j}\right\|^{2}\right)^{1 / 2} .
\end{array}
$$

The space $X$ is said to be of Gaussian type $p$ (resp. Gaussian cotype $q$ ) if $T_{p}(X)=\sup _{n} T_{p, n}(X)<$ $\infty\left(\right.$ resp. $\left.C_{q}(X)=\sup _{n} C_{q, n}(X)<\infty\right)$. One can analogously define the Rademacher type and cotype by exchanging the Gaussian sequence by a Rademacher sequence. The results shown in this note are valid for both notions of type and cotype.

For $r \in[1, \infty)$, we denote by $S_{r}^{d}$ the Hermitian part of the $d$-dimensional $r$-Schatten class and by $l_{r}^{d}$ the classical space of $r$-summable sequences in $\mathbb{R}^{d}$; the space $S_{r}^{d}$ is a real Banach space with norm $\|x\|_{r}:=\left(\operatorname{Tr}|x|^{r}\right)^{1 / r}$. Table 1 summarizes the behaviour of the type and cotype constants for the $r$-Schatten classes that we use (see Ref. [9] for details).

We now introduce some notation. The set of rank-one projections in $\mathbb{C}^{d \times d}$ is denoted by $\mathbb{P}\left(\mathbb{C}^{d}\right)$. The unit ball of a space $Z$ is written as $B_{Z}$. The convex hull of a set $S$ is the set of convex combinations of elements of $S$, which we denote by conv $(S)$. The set of linear maps between $X$ and $Y$ is $L(X, Y)$. A linear projection $P \in L(X, Y)$ is a linear map such that $P^{2}=P$. Finally, we denote by $\langle x, y\rangle:=\operatorname{Tr}(x y)$ the Hilbert-Schmidt inner product in the real vector space of Hermitian matrices $\mathcal{H}_{d}$.

In the next section, we introduce a special space which will generate the linear approximation to the almost-linear map $F: X \rightarrow Y$. This space is an extension of $X$ and $Y$ and is called a twisted sum (basically because it "twists" the unit ball of $X$ and $Y$ according to $F$ ). Twisted sums were extensively studied by Kalton [1] in the context of the three-space problem. In particular, Kalton showed that twisted sums are in correspondence with quasi-linear maps; this is a weaker condition than almost-linearity, 
but, for our purposes, it suffices to say that any almost-linear map is a quasi-linear map. See Ref. [10] for a detailed exposition of this topic.

Table 1. Upper bounds for the Rademacher type and cotype constants of the spaces $l_{r}^{d}$ and $S_{r}^{d}$. The Gaussian type and cotype for these spaces behave in the same way, up to a factor of $\sqrt{2 / \pi}$, as the Rademacher type and cotype. For a Hilbert space, the type and cotype constants are always equal to one.

\begin{tabular}{ccc}
\hline & Type $p \in[\mathbf{1}, \mathbf{2}]$ & Cotype $q \in[\mathbf{2 , \infty}]$ \\
\hline$l_{1}^{d}$ & $d^{1-\frac{1}{p}}$ & $\sqrt{2}$ \\
Hilbert space & 1 & 1 \\
$l_{\infty}^{d}$ & $(4 \log d)^{1-1 / p}$ & $d^{1 / q}$ \\
$S_{1}^{d}$ & $d^{1-1 / p}$ & $\sqrt{e}$ \\
$S_{\infty}^{d}$ & $(4 \log d)^{1-1 / p}$ & $d^{1 / q}$ \\
\hline
\end{tabular}

\section{Finite-Dimensional Twisted Sums}

Let $X, Y$ be two Banach spaces with dimension $d_{1}, d_{2}$, respectively. The twisted sum of $Y$ and $X$ is a $\left(d_{1}+d_{2}\right)$-dimensional space $Z$ that contains a subspace $Y_{0}$ that is isomorphic to $Y$ and such that $Z / Y_{0}$ is isomorphic to $X$. The twisted sums that interest us are constructed with an almost-linear function $F$. Consider $\delta>0$ and the Cartesian product $Y \oplus X$ (the order is important) endowed with the quasi-norm:

$$
\|(y, x)\|_{F}:=\frac{\|y-F(x)\|_{Y}}{\delta}+\|x\|_{X} .
$$

Then, $Y_{0}=\{(y, 0): y \in Y\}$ is $\delta^{-1}$-isometric to $Y$ and $Z / Y_{0}$-isometric to $X$. Note that, since $F$ is homogeneous, $\|(-y,-x)\|_{F}=\|\|(y, x) \|_{F}$ and $\|(y, x)\|_{F}=0$ implies that $(y, x)=0$. Although $\left\|\left(y_{1}, x_{1}\right)+\left(y_{2}, x_{2}\right)\right\|_{F} \leq 2\left(\|\|\left(y_{1}, x_{1}\right)\left\|_{F}+\right\|\left(y_{2}, x_{2}\right) \|_{F}\right)$, we can still endow $Z$ with a norm. The twisted sum $Z$ can be made into a Banach space with the norm

$$
\|(y, x)\|:=\inf \left\{\sum_{j}\left\|\left(y_{j}, x_{j}\right)\right\|_{F}:(y, x)=\sum_{j}\left(y_{j}, x_{j}\right)\right\} .
$$

The fact that the above expression defines a norm will be shown below. The completion of a quasi-Banach space $Z$ whose dual is non-trivial with respect to this norm is known as the Banach envelope of $Z$ [11]. In order to avoid charged notation, we also denote the Banach envelope by $Z$.

Lemma 1. Let $\||\cdot| \mid$ be a quasi-norm on $Z$, then the following equivalent expressions define a norm on $Z$. For $z \in Z$,

$$
\begin{aligned}
\|z\| & =\inf \left\{\sum_{j=1}^{n}\left\|z_{j}\right\|: z=\sum_{j=1}^{n} z_{j}\right\}, \\
& =\inf \left\{\lambda>0: z / \lambda \in \operatorname{conv}\left(B_{Z}\right)\right\}, \\
& =\inf \left\{\xi(z): \xi \in Z^{*},\|\xi\| \leq 1\right\} .
\end{aligned}
$$

Moreover, for the quasi-norm defined by Equation (3), we have the following equivalence:

$$
\|(y, x)\| \leq\|(y, x)\|_{F} \leq 2\|(y, x)\| .
$$

Proof. We show first that the first expression indeed defines a norm. Since \|\|$\cdot \|$ is a quasi-norm, the only property that we need to check is the triangle inequality. This can be verified by 


$$
\begin{aligned}
\left\|z_{1}+z_{2}\right\| & =\inf \left\{\sum_{j=1}^{n} \mid\left\|w_{j}\right\|: z_{1}+z_{2}=\sum_{j=1}^{n} w_{j}=\sum_{j=1}^{n_{1}} w_{j}+\sum_{j=1}^{n_{2}} w_{j}\right\}, \\
& \leq \inf \left\{\sum_{j=1}^{n_{1}}\left\|w_{j}\right\|: z_{1}=\sum_{j=1}^{n_{1}} w_{j}\right\}+\inf \left\{\sum_{j=1}^{n_{2}}\left\|w_{j}\right\|: z_{2}=\sum_{j=1}^{n_{2}} w_{j}\right\}, \\
& =\left\|z_{1}\right\|+\left\|z_{2}\right\|,
\end{aligned}
$$

as those are valid decompositions of $z_{1}+z_{2}$. We show now that Equations (5) and (6) are the same. Let $\alpha=\|z\|$ be the infimum of Equation (6). Then, there exist $m \in \mathbb{N}$, positive real numbers $\left(\lambda_{j}\right)_{j=1}^{m}$, $\sum_{j=1}^{m} \lambda_{j}=1$ and $\left(z_{j}\right)_{j=1}^{m}$ with quasi-norm one such $z=\alpha \sum_{j=1}^{m} \lambda_{j} z_{j}$. This is a valid decomposition of $z$ and $\sum_{j=1}^{m}|| \alpha \lambda_{j} z_{j} \| \mid \leq \alpha$. On the other hand, let $z=\sum_{j=1}^{m} z_{j}$ be the decomposition that achieves the infimum in Equation (5) so that $\|z\|=\sum_{j=1}^{m} \mid\left\|z_{j}\right\|$. . Then,

$$
\frac{z}{\sum_{k=1}^{m}\left\|z_{k}\right\|}=\sum_{j=1}\left(\frac{\left\|z_{j}\right\| \|}{\sum_{k=1}^{m}\left\|z_{k}\right\| \mid}\right) \frac{z_{j}}{\left\|z_{j}\right\|} \in \operatorname{conv}\left(B_{Z}\right) .
$$

The norm of $\xi \in Z^{*}$ can be computed as

$$
\|\xi\|=\sup _{z \in \operatorname{conv}\left(B_{Z}\right)}|\xi(z)|=\sup _{z \in B_{Z}}|\xi(z)|=\sup \{|\xi(z)|:\|z \mid\| \leq 1\},
$$

as the supremum over a convex function is achieved at the extremal points. Thus, the dual of the quasi-Banach space $Z$ and its Banach envelope coincide. Thus, Equation (7) is just the usual expression in terms of the dual. We now compare the quasi-norm in Equation (3) with the norm of its envelope.

Since $\|z\|$ is defined by the infimum of $\sum_{j}\left|\left\|z_{j}\right\|\right|$ over all the decompositions of $z$, Equation (5), we immediately have the first inequality in Equation (8). For the second inequality, let $(y, x)=$ $\sum_{j}\left(y_{j}, x_{j}\right)$, then, using Equation (2),

$$
\begin{aligned}
\|(y, x)\|_{F} & =\frac{\|F(x)-y\|_{Y}}{\delta}+\|x\|_{X}, \\
& =\frac{\left\|F\left(\sum_{j} x_{j}\right)-\sum_{j} F\left(x_{j}\right)+\sum_{j} F\left(x_{j}\right)-\sum_{j} y_{j}\right\|_{Y}}{\delta}+\left\|\sum_{j} x_{j}\right\|_{X}, \\
& \leq \frac{\left\|F\left(\sum_{j} x_{j}\right)-\sum_{j} F\left(x_{j}\right)\right\|_{Y}}{\delta}+\sum_{j} \frac{\left\|F\left(x_{j}\right)-y_{j}\right\|_{Y}}{\delta}+\sum_{j}\left\|x_{j}\right\|_{X^{\prime}} \\
& \leq \frac{\delta \sum_{j}\left\|x_{j}\right\|_{X}}{\delta}+\sum_{j} \frac{\left\|F\left(x_{j}\right)-y_{j}\right\|_{Y}}{\delta}+\sum_{j}\left\|x_{j}\right\|_{X^{\prime}} \\
& \leq 2 \sum_{j}\left\|\left(y_{j}, x_{j}\right)\right\| \|_{F} .
\end{aligned}
$$

Additionally, we can understand the resulting twisted sum $Z$ with a norm as in Equation (5) as the space with unit ball [12]

$$
B_{Z}:=\operatorname{conv}\left(\left\{(y, 0):\|y\|_{Y} \leq 1\right\} \cup\left\{(F(x), x):\|x\|_{X} \leq 1\right\}\right) .
$$

We write $Z=Y \oplus_{F} X$ for the (Banach envelope of) twisted sum of $Y$ and $X$ generated by the almost-linear map $F: X \rightarrow Y$. 


\section{Main Result}

We are now ready to put all the pieces together and to make the connection explicitly between (co)type constants and the linear stability of almost-linear maps.

Theorem 1. Let $F: X \rightarrow Y$ be an almost-linear map between finite-dimensional real Banach spaces, i.e., $F$ is a real homogeneous map and there exists a $\delta>0$ such that, for any finite sequence $\left(x_{i}\right)_{i=1}^{m} \subset X, m \in \mathbb{N}$ and $\lambda \in \mathbb{R}^{m}$,

$$
\left\|\sum_{i=1}^{m} \lambda_{i} F\left(x_{i}\right)-F\left(\sum_{i=1}^{m} \lambda_{i} x_{i}\right)\right\|_{Y} \leq \delta \sum_{i=1}^{m}\left|\lambda_{i}\right|\left\|x_{i}\right\|_{X} .
$$

Let $Z=Y \oplus_{F} X$ be the respective twisted sum generated by this map. Then,

$$
\inf _{H \in L(X, Y)} \sup _{x \in X} \frac{\|F(x)-H(x)\|_{Y}}{\|x\|_{X}} \leq 2 \delta \min \left\{T_{2}(Z) C_{2}(X), 1+T_{2}\left(Z^{*}\right) C_{2}\left(Y^{*}\right)\right\},
$$

where $T_{2}$ and $C_{2}$ are the type 2 and cotype 2 constants.

Proof of Theorem 1. We need the following important theorem of Maurey [13] (see Theorem 7.4.4 in Ref. [14] for a modern proof).

Theorem 2 (Maurey's Extension). Let $E$ be a Banach space and $S$ a closed subspace of $E$. Let $T_{2}(E)$ be either the Gaussian or Rademacher type 2 constant of $E$ and $C_{2}(S)$ either the Gaussian or Rademacher cotype 2 constant of $S$. Then, there exists a projection $P: E \rightarrow S$ with

$$
\|P\| \leq T_{2}(E) C_{2}(S) .
$$

We remark that the norm $\|\cdot\|$ in Theorem 2 is the operator norm. This might seem odd at first sight as usually the projections are considered between Hilbert spaces and in that case they always have a norm equal to one. This is no longer true when we leave the special world of Hilbert spaces and consider general Banach spaces. Maurey's theorem is proven by factorizing through a Hilbert space though. In a sense, the notions of type and cotype measure how far we are from the Hilbert space scenario.

Let $Z=Y \oplus_{F} X$ be the twisted sums of $Y$ and $X$ and consider the Banach envelope of $Z$. Let us denote by $Z$ as well the Banach envelope of $Z$. From Maurey's theorem, we know there exists a projection $P: Z \rightarrow X$ such that $\|P\| \leq T_{2}(Z) C_{2}(X)$. Since $P$ is a projection, it has the general form $P(y, x)=(y-H(x), 0)$, where $H: X \rightarrow Y$ is a linear map. Then, using Equation (8),

$$
\begin{aligned}
\|P\|=\sup _{(y, x) \in Z} \frac{\|P(y, x)\|}{\|(y, x)\|} & \geq \sup _{x \in X} \frac{\|P(F(x), x)\|}{\|(F(x), x)\|} \\
& =\sup _{x \in X} \frac{\|(F(x)-H(x), 0)\|}{\|(F(x), x)\|} \\
& \geq \sup _{x \in X} \frac{\|(F(x)-H(x), 0)\|}{2\|(F(x), x)\|} \\
& =\sup _{x \in X} \frac{\|F(x)-H(x)\|_{Y}}{2 \delta\|x\|_{X}} \\
& \geq \inf _{H \in L(X, Y)} \sup _{x \in X} \frac{\|F(x)-H(x)\|_{Y}}{2 \delta\|x\|_{X}} .
\end{aligned}
$$

We can also consider a dual construction for a different bound. Let $Z^{*}$ be the dual of the twisted sum $Y \oplus_{F} X$. It is known [15] that the dual of $Z$ is isomorphic to $X^{*} \oplus_{F^{*}} Y^{*}$, where $F^{*}$ is in some sense 
the dual map of $F$ (see [15] for details). Since we are dealing with finite-dimensional spaces, $Z^{* *}$ can be identified with $Z$. Let $Q: Z^{*} \rightarrow Y^{*}$ be the projection obtained by Maurey's extension theorem when applied to the Banach spaces $Z^{*}$ and $Y^{*}$. Let us consider the projection $\tilde{P}: Z \rightarrow X$ defined via $\tilde{P}:=\mathrm{id}-Q^{*} \pi$, where $\pi$ is the quotient map $\pi: Z \rightarrow X, \pi(y, x)=x$. Indeed, let $\Omega: X \rightarrow Y$ be the linear map induced by $Q^{*}$. Then,

$$
\begin{aligned}
\tilde{P}(y, x) & =(y, x)-Q^{*} \pi(y, x) \\
& =(y, x)-Q^{*} x \\
& =(y, x)-(\Omega(x), x)=(y-\Omega(x), 0) \in X .
\end{aligned}
$$

Analogously with the previous calculation, we find

$$
\inf _{\Omega \in L(X, Y)} \sup _{x \in X} \frac{\|F(x)-\Omega(x)\|_{Y}}{\|x\|_{X}} \leq 2 \delta\|\tilde{P}\| \leq 2 \delta(1+\|Q\|) .
$$

The final results then follows from the upper bound that Maurey's theorem provides on the norm of such projections.

\section{Applications}

The following result gives an improvement on Theorem 2-(ii) in [8].

Theorem 3 (Linear Stability of Wigner's theorem). Let $f: \mathbb{P}\left(\mathbb{C}^{d}\right) \rightarrow \mathbb{P}\left(\mathbb{C}^{d}\right)$ be a function that satisfies

$$
|\langle f(x), f(y)\rangle-\langle x, y\rangle| \leq \varepsilon \quad \text { for all } x, y \in \mathbb{P}\left(\mathbb{C}^{d}\right) .
$$

Then, there exists a universal constant $C$ and a linear map $H: \mathcal{H}_{d} \rightarrow \mathcal{H}_{d}$ such that, for all $x \in \mathbb{P}\left(\mathbb{C}^{d}\right)$,

$$
\|f(x)-H(x)\|_{2} \leq\left(C \log _{2} d\right)^{\beta} \sqrt{d \varepsilon},
$$

where $\beta=2+\frac{1}{2} \log _{2} \log _{2} 2 d$.

We call a map $f: \mathbb{P}\left(\mathbb{C}^{d}\right) \rightarrow \mathbb{P}\left(\mathbb{C}^{d}\right)$ that satisfies Equation (10) an almost-symmetry. In order to prove Theorem 3, we make use of the following lemmas (c.f. Theorem 1 in [12]). First, we need the type constant of a twisted sum (cf. Lemma 16.6-7 in [16]).

Lemma 2. Let $Z$ be the twisted sum of $Y$ and $X$; then,

$$
T_{2, n^{2}}(Z) \leq T_{2, n}(Y) T_{2, n}(Z)+T_{2, n}(Y) T_{2, n}(X)+T_{2, n}(Z) T_{2, n}(X)
$$

The type 2 constant of a Banach space of dimension $d$ can be obtained from the type constant restricted to families of size $d(d+1) / 2$ as stated by the following lemma. This result follows from a cone version of Caratheodory's theorem (see Lemma 6.1 in [17]).

Lemma 3. Let $X$ be a d-dimensional Banach space. Then, $T_{2, n}(X)=T_{2, d(d+1) / 2}(X)$ and $C_{2, n}(X)=$ $C_{2, d(d+1) / 2}(X)$ for any $n \geq d(d+1) / 2$.

Proof. The first step of the proof consists of extending the function $f$ to $F: S_{1}^{d} \rightarrow S_{1}^{d}$ such that $\left.F\right|_{\mathbb{P}\left(\mathbb{C}^{d}\right)}=f$. We take $x$ in the unit sphere of $S_{1}^{d}$ and identify it with its antipodal point $-x$. We choose a fixed spectral decomposition for both elements, say $x=\sum_{j=1}^{d} \lambda_{j} x_{j}$, and define $F(x):=\sum_{j=1}^{d} \lambda_{j} f\left(x_{j}\right)$. Then, we can extend $F$ homogeneously from the unit sphere to any $y \in S_{1}^{d}$ by multiplying $x$ or $-x$ with $\lambda \geq 0$ so that $\lambda x=y$ or $-\lambda x=y$. We call again this extension $F$. By construction, $F$ is a real homogeneous map. Note that this extension is not unique, but we do not need this here. 
As proven in Lemma 2 of Ref. [8], $F$ is an almost-linear map

$$
\left\|\sum_{i=1}^{m} \lambda_{i} F\left(x_{i}\right)-F\left(\sum_{i=1}^{m} \lambda_{i} x_{i}\right)\right\|_{2} \leq \delta \sum_{i=1}^{m}\left|\lambda_{i}\right|\left\|x_{i}\right\|_{1},
$$

with $\delta=2 \sqrt{\varepsilon}$. If we use Theorem 1 with the twisted sum $Z:=S_{2}^{d} \oplus_{F} S_{1}^{d}$, we will see that we cannot obtain anything better than a linear dependence on $d$. However, we will be able to obtain a better dimension dependence if we consider a dual construction, namely with $Z^{*}:=S_{\infty}^{d} \oplus_{F^{*}} S_{2}^{d}$. For that matter, we use Lemmas 2 and 3 in order to estimate the type 2 constant of $Z^{*}$. From Equation (11) and $T_{2}\left(S_{\infty}\right) \leq \sqrt{4 \log d}$, we obtain $T_{2, n^{2}}\left(Z^{*}\right) \leq 2 \sqrt{8 \log _{2} d} T_{2, n}\left(Z^{*}\right)$ for all $n \in \mathbb{N}$. It is known that, for a general Banach space $E, T_{2}(E) \leq \sqrt{\operatorname{dim}(E)}$ (Proposition 12.3 in [9]). Thus, for all two-dimensional subspaces of $Z$, the type is less than $\sqrt{2}$ and $T_{2,2}(Z) \leq \sqrt{2}$ (this can be alternatively derived from a classical result of John and the relation between the Banach-Mazur distance and type 2 constants). It follows from induction that

$$
T_{2,2^{2^{k}}}\left(Z^{*}\right) \leq\left(2 \sqrt{8 \log _{2} d}\right)^{k} \sqrt{2}
$$

which, in turn, implies

$$
T_{2, n}\left(Z^{*}\right) \leq \sqrt{2}\left(\log _{2} n\right)\left(8 \log _{2} d\right)^{\frac{\log _{2} \log _{2} n}{2}} .
$$

The dimension of the real vector space of Hermitian matrices $\mathcal{H}_{d}$ is $d^{2}$. Therefore, we obtain from Lemma 3 with $n=2 d^{4}$

$$
T_{2}\left(Z^{*}\right) \leq 2\left(8 \log _{2} d\right)^{2+\frac{\log _{2} \log _{2} 2 d}{2}} .
$$

It follows from Theorem 1 and $C_{2}\left(S_{\infty}^{d}\right) \leq \sqrt{d}$ that there exists a linear map $H: S_{1}^{d} \rightarrow S_{2}^{d}$ such that

$$
\sup _{x \in \mathbb{P}\left(\mathbb{C}^{d}\right)}\|f(x)-H(x)\|_{2} \leq 4\left(8 \log _{2} d\right)^{2+\frac{\log _{2} \log _{2} 2 d}{2}} \sqrt{d \varepsilon} .
$$

The following proposition is essentially due to Kalton. It can be shown using Theorem 2.2 in [3] as $S_{2}^{d}$ and $\mathbb{R}^{d^{2}}$ are isomorphic Hilbert-spaces. We present here a proof using the notions of (co)type and Theorem 1 .

Proposition 1 (Stability of Global Symmetries). Let $f: B_{S_{2}^{d}} \rightarrow S_{2}^{d}$ be a continuous function that satisfies

$$
|\langle f(x), f(y)\rangle-\langle x, y\rangle| \leq \varepsilon \quad \text { for all } x, y \in B_{S_{2}^{d}}
$$

Then, there exists a linear map $H: S_{2}^{d} \rightarrow S_{2}^{d}$ and an absolute constant $C$ such that, for all $X \in B_{S_{2}^{d}}$,

$$
\|f(x)-H(x)\|_{2} \leq C \sqrt{\varepsilon} \log _{2} d .
$$

Proof of Proposition 1. The first step consists of showing that the function $f$ can be extended to a continuous homogeneous function on the whole space without paying much.

Lemma 4. Let $f: B_{S_{2}^{d}} \rightarrow S_{2}^{d}$ be a continuous function that satisfies

$$
|\langle f(x), f(y)\rangle-\langle x, y\rangle| \leq \varepsilon \quad \text { for all } x, y \in B_{S_{2}^{d}} .
$$


Then, there exists a continuous and homogeneous function $F: S_{2}^{d} \rightarrow S_{2}^{d}$ such that

$$
\left\|\sum_{j=1}^{n} F\left(x_{j}\right)-F\left(\sum_{j=1}^{n} x_{j}\right)\right\|_{2} \leq 4 \sqrt{\varepsilon} \sum_{j=1}^{n}\left\|x_{j}\right\|_{2} \quad \text { for all } x_{j} \in S_{2}^{d},
$$

and

$$
\sup _{X \in B_{S_{2}^{d}}}\|f(x)-F(x)\|_{2} \leq 3 \sqrt{\varepsilon}
$$

Proof of Lemma 4. Let us extend $f$ to $F: S_{2}^{d} \rightarrow S_{2}^{d}$ where

$$
F(x):=\|x\|_{2}\left(f\left(\frac{x}{2\|x\|_{2}}\right)-f\left(-\frac{x}{2\|x\|_{2}}\right)\right) .
$$

This function is homogeneous, i.e., $F(\lambda x)=\lambda F(x)$ for all $\lambda \in \mathbb{R}$, and continuous as $f$ and $\|\cdot\|$ are also continuous. Using Equation (12) and the triangle inequality, we obtain the new almost-symmetry condition

$$
|\langle F(x), F(y)\rangle-\langle x, y\rangle| \leq 4 \varepsilon\|x\|_{2}\|y\|_{2} .
$$

Hence, for any $z \in S_{2}^{d}$,

$$
\begin{aligned}
\left|\left\langle\sum_{j=1}^{n} F\left(x_{j}\right)-F\left(\sum_{j=1}^{n} x_{j}\right), F(z)\right\rangle\right| & =\left|\left\langle\sum_{j=1}^{n} F\left(x_{j}\right)-F\left(\sum_{j=1}^{n} x_{j}\right), F(z)\right\rangle-\left\langle\sum_{j=1}^{n} x_{j}-\sum_{j=1}^{n} x_{j}, z\right\rangle\right| \\
& \leq 8 \varepsilon \sum_{j=1}^{n}\left\|x_{j}\right\|_{2}\|z\|_{2} .
\end{aligned}
$$

Therefore, from the linearity of the inner product, we obtain Equation (13). Finally, we show that $f$ and $F$ are $\sqrt{\varepsilon}$-close. From Equation (12),

$$
\begin{aligned}
& |\langle F(x), f(x)\rangle-\langle x, x\rangle| \\
& =\left|\|x\|_{2}\left(\left\langle f\left(\frac{x}{2\|x\|}\right), f(x)\right\rangle-\frac{\|x\|_{2}}{2}\right)-\|x\|_{2}\left(\left\langle f\left(\frac{-x}{2\|x\|}\right), f(x)\right\rangle+\frac{\|x\|_{2}}{2}\right)\right| \\
& \leq 2 \varepsilon\|x\|_{2} .
\end{aligned}
$$

Thus, with Equation (15), we have

$$
\begin{aligned}
\|F(x)-f(x)\|_{2}^{2} & =\|F(x)\|_{2}^{2}-\|x\|_{2}^{2}-2 \operatorname{Re}(\langle F(x), f(x)\rangle-\langle x, x\rangle)+\|f(x)\|_{2}^{2}-\|x\|_{2}^{2} \\
& \leq 4 \varepsilon\|x\|_{2}^{2}+4 \varepsilon\|x\|_{2}+\varepsilon
\end{aligned}
$$

which is less than $9 \varepsilon$ for all $x \in B_{S_{2}^{d}}$.

We consider now the twisted sum $Z=S_{2}^{d} \oplus_{F} S_{2}^{d}$ generated by the almost-linear map $F$. Before applying Theorem 1, we estimate the type 2 constant of $Z$. Since $S_{2}^{d}$ is a Hilbert space, it has a type 2 constant equal to one and we obtain from Lemma 2 that

$$
T_{2, n^{2}}(Z) \leq 1+2 T_{2, n}(Z) \quad \text { for all } n
$$


As in the proof of Theorem 3, all two-dimensional subspaces of $Z$ have a type less than $\sqrt{2}$ and $T_{2,2}(Z) \leq \sqrt{2}$. It follows from induction that, for $n \geq 3$,

$$
T_{2, n}(Z) \leq 2(1+\sqrt{2}) \log _{2} n
$$

Hence, from Lemma 3 with $n=4 d^{2}$,

$$
T_{2}(Z) \leq 4(1+\sqrt{2}) \log _{2} 2 d .
$$

Accordingly, from $C_{2}\left(S_{2}^{d}\right)=1$ and Theorem 1, there exists a linear map $H: S_{2}^{d} \rightarrow S_{2}^{d}$ such that, for all $x \in B_{S_{2}^{d}}$,

$$
\|F(x)-H(x)\|_{2} \leq 32(1+\sqrt{2}) \log _{2}(2 d) \sqrt{\varepsilon} .
$$

Finally, from Equation (14) and the triangle inequality, we obtain

$$
\begin{aligned}
\sup _{x \in B_{S_{2}^{d}}}\|f(x)-H(x)\|_{2} & \leq \sup _{x \in B_{S_{2}^{d}}}\|f(x)-F(x)\|_{2}+\|F(x)-H(x)\|_{2} \\
& \leq 79 \sqrt{\varepsilon}\left(1+\log _{2} d\right) .
\end{aligned}
$$

\section{Discussion and Perspectives}

Using Theorem 1, we are able to improve-up to some logarithmic factors-the upper bound on the dimension dependence of the linear stability of Wigner's theorem from $d$ to $\sqrt{d}$. There seems to be room for an exponential improvement in the dimension as the lower bound is of order $\log d$ (see the discussion section of [8]). The method developed here allows us to study systematically the limitations of considering other types of almost-linear extensions to solve this problem. Even if we were able to extend the almost-symmetry $f$ to an almost-linear map $F: S_{1}^{d} \rightarrow S_{1}^{d}$ with $\delta$ independent of $d$, we would still get from Theorem 1 an upper bound of order $\sqrt{d}$. This is just a consequence of how the type and cotype constants of $S_{1}^{d}$ and $S_{\infty}^{d}$ behave. There is a trade-off in Theorem 1 between the type constant for individual spaces and the type constant of their twisted sum.

It can be seen from Table 1 and Lemma 2 that the best bound that can be obtained from Theorem 1 is in the case that $X$ and $Y$ are Hilbert spaces. This is the case of Proposition 1 and a logarithmic dependence is obtained there. However, the almost-symmetry condition holds there for the entire Hilbert-Schmidt unit ball, while, in Wigner's theorem, the almost-symmetry condition is required to hold only for the non-convex space of normalized hermitian rank-one projections.

Funding: This research received no external funding.

Acknowledgments: The author would like to thank Marius Junge, Willian Corrêa and Cambyse Rouzé for valuable discussions. Furthermore, the author wishes to thank the Institut Henri Poincaré in Paris and the organizers of the trimester on "Analysis in Quantum Information Theory" (IHP17). This work was supported by the German Research Foundation (DFG) and the Technical University of Munich (TUM) in the framework of the Open Access Publishing Program.

Conflicts of Interest: The authors declare no conflicts of interest.

\section{References}

1. Kalton, N.J.; Peck, N.T. Twisted sums of sequence spaces and the three space problem. Trans. Amer. Math. Soc. 1979, 255, 1-30. [CrossRef]

2. Brudnyi, Y.; Kalton, N.J. Polynomial approximation on convex subsets of $\mathbb{R}^{n}$. Constr. Approx. 2000, 16, 161-200. [CrossRef]

3. Kalton, N.J. A remark on quasi-isometries. Proc. Am. Math. Soc. 2003, 131, 1225-1231. [CrossRef] 
4. Jung, S.-M. Hyers-Ulam-Rassias Stability of Functional Equations in Nonlinear Analysis; Springer: New York, NY, USA, 2011; Volume 48.

5. Wigner, E.P. Gruppentheorie und ihre Anwendung auf die Quantenmechanik der Atomspektren; Fredrik Vieweg und Sohn: Braunschweig, Germany, 1931; pp. 233-236.

6. Molnár, L.; Nagy, G.; Szokol, P. Maps on density operators preserving quantum $f$-divergences. Quantum Inf. Process. 2013, 12, 2309-2323. [CrossRef]

7. Aubrun, G.; Szarek, S.J. Alice and Bob Meet Banach: The Interface of Asymptotic Geometric Analysis and Quantum Information Theory; American Mathematical Society: Providence, RI, USA, 2017; Volume 223.

8. Cuesta, J.; Wolf, M.M. Are almost-symmetries almost linear? J. Math. Phys. 2019, 60, 082101. [CrossRef]

9. Tomczak-Jaegermann, N. Banach-Mazur Distances and Finite-Dimensional Operator Ideals; Longman Scientific \& Technical: Harlow, UK, 1989.

10. Castillo, J.M.F.; González, M. Three-Space Problems in Banach Space Theory; Springer: Berlin/Heidelberg, Germany, 1997.

11. Kalton, N.J.; Peck, N.T.; Roberts, J. An F-Space Sampler; Cambridge University Press: Cambridge, UK, 1984; Volume 89.

12. Enflo, P.; Lindenstrauss, J.; Pisier, G. On the three space problem. Math. Scand. 1975, 36, 199-210. [CrossRef]

13. Maurey, B. Un théorème de prolongement. C. R. Acad. Sc. Paris 1974, 279, 329-332.

14. Albiac, F.; Kalton, N.J. Topics in Banach Space Theory, 1st ed.; Springer: New York, NY, USA, 2006.

15. Cabello Sánchez, F.; Castillo, J.M.F. Duality and twisted sums of Banach spaces. J. Funct. Anal. 2000, 175, 1-16. [CrossRef]

16. Benyamini, Y.; Lindenstrauss, J. Geometric Nonlinear Functional Analysis; American Mathematical Society: Providence, RI, USA, 2000; Volume 1.

17. Figiel, T.; Lindenstrauss, J.; Milman, V.D. The dimension of almost spherical sections of convex bodies. Acta Math. 1977, 139, 53-94. [CrossRef]

(C) 2019 by the author. Licensee MDPI, Basel, Switzerland. This article is an open access article distributed under the terms and conditions of the Creative Commons Attribution (CC BY) license (http:/ / creativecommons.org/licenses/by/4.0/). 